\title{
Regulation of AMPA receptor endocytosis by a signaling mechanism shared with LTD
}

\author{
Eric C. Beattie ${ }^{2}$, Reed C. Carroll ${ }^{1}$, Xiang Yu ${ }^{1}$, Wade Morishita ${ }^{1}$, Hiroki Yasuda ${ }^{1}$, \\ Mark von Zastrow ${ }^{2}$ and Robert C. Malenka ${ }^{1}$

\footnotetext{
${ }^{1}$ Nancy Pritzker Laboratory, Department of Psychiatry and Behavioral Sciences, Stanford University School of Medicine, Stanford, California 94304, USA

2 Departments of Psychiatry and Cellular and Molecular Pharmacology, University of California, San Francisco, California 94143, USA

The first two authors contributed equally to this work

Correspondence should be addressed to R.C.M. (malenka@stanford.edu)
}

\begin{abstract}
The endocytosis of AM PA receptors is thought to be important in the expression of long-term depression (LTD) triggered by NM DA receptor activation. Although signaling pathways necessary for LTD induction have been identified, those responsible for the regulated internalization of AM PA receptors are unknown. Here we show that activation of NMDA receptors alone can trigger AMPA receptor endocytosis through calcium influx and activation of the calcium-dependent protein phosphatase calcineurin. A distinct signaling mechanism mediates the AM PA receptor endocytosis stimulated by insulin. These results demonstrate that although multiple signaling pathways can induce AM PA receptor internalization, NMDA receptor activation enhances AM PA receptor endocytosis via a signaling mechanism required for the induction of LTD.
\end{abstract}

Over the last five years, evidence has accumulated that NMDAreceptor-dependent long-term potentiation (LTP) and LTD involve cycling of AMPA receptors (AMPARs) into and out of the synaptic plasma membrane, respectively ${ }^{1-3}$. The initial motivation for pursuing this idea derived from studies suggesting that a proportion of excitatory synapses in the hippocampus are functionally silent at resting membrane potentials because they contain NMDA receptors (NMDARs) but not AMPARs, and that LTP involves the appearance of AMPAR-mediated responses at these synapses ${ }^{4-7}$. Although these electrophysiological observations were replicated in a number of preparations (for review, see refs. 3, 8), alternative explanations that do not require the existence of silent synapses have been presented ${ }^{9-11}$. Thus it has been important to take complementary approaches to understanding the role of AMPAR trafficking in synaptic plasticity, the most important of which involves visualizing AMPARs and NMDARs and determining whether activity affects their synaptic distribution.

Neural activity can indeed differentially regulate the synaptic distribution of AMPARs and NMDARs, as initially demonstrated with prolonged pharmacological manipulations of activity (hours to days) in cultured neurons ${ }^{12-14}$. Soon thereafter it was shown that brief application of glutamate causes a rapid loss of AMPARs, but not NMDARs, from synaptic sites ${ }^{15}$, and that synaptically evoked LTD is accompanied by a loss of surface, synaptic AMPARs ${ }^{16}$. Furthermore, LTP involves the delivery of AMPARs to synapses, as was shown by directly visualizing a fusion protein consisting of GFP and the AMPAR subunit GluR1 (ref. 17).

What signal transduction mechanisms control the gain and loss of AMPARs during LTP and LTD, respectively? Consistent with an important role for CaMKII in the triggering of $\mathrm{LTP}^{1-3}$, the delivery of recombinant AMPARs to the synaptic plasma membrane is greatly enhanced by increasing CaMKII activity ${ }^{18}$. How this delivery actually occurs, however, remains unknown, although one prominent possibility is that it involves membrane fusion and exocytotic events ${ }^{19}$.

Progress in understanding the mechanisms underlying LTD also has proceeded rapidly. AMPARs undergo dynamin- and clathrin-dependent endocytosis, which is necessary for LTD in both hippocampal neurons and cerebellar Purkinje cells ${ }^{20-23}$. The link between AMPAR endocytosis and LTD, however, is complicated by the apparently complex nature of the mechanisms regulating AMPAR internalization. Although synaptically released glutamate can induce AMPAR endocytosis ${ }^{15,20}$, this trafficking event also is triggered by an insulin-activated signaling pathway that interacts with, but is unlikely to be directly involved in, the induction of $\mathrm{LTD}^{23}$.

Thus a critical question is whether, like NMDAR-dependent LTD in the hippocampus, NMDAR activation can lead to the triggering of AMPAR endocytosis, and if so, what signal transduction pathway mediates this effect? The most prominent hypothesis for the triggering of NMDAR-dependent LTD proposes that LTD requires a modest rise in postsynaptic calcium that preferentially activates a protein phosphatase cascade ${ }^{24-27}$. Here we present evidence that the NMDAR-dependent activation of a similar signal transduction pathway regulates AMPAR endocytosis. Surprisingly, however, the actions of insulin on AMPAR trafficking seem to be mediated by an independent signaling mechanism, indicating that AMPAR endocytosis can be triggered by multiple signaling pathways, only some of which are activated during LTD. 
a
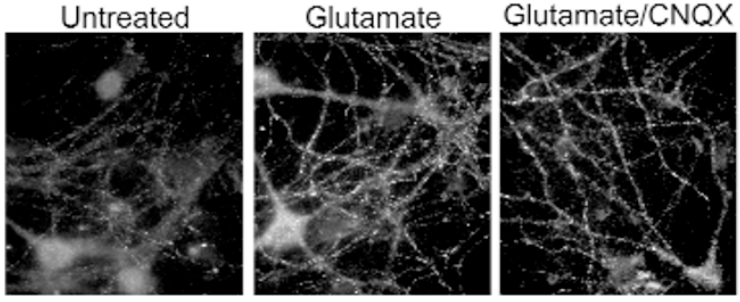

C

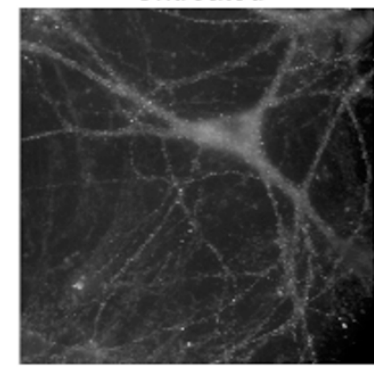

NMDA-treated

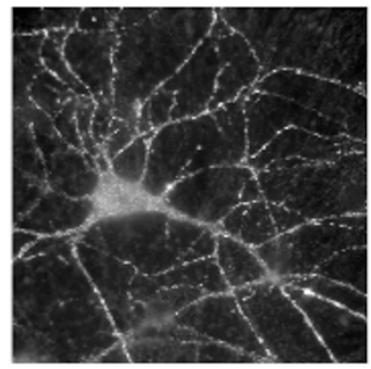

b

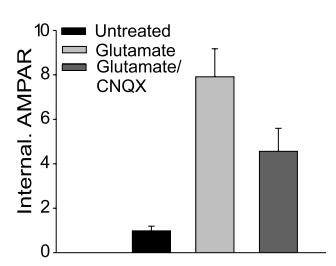

d

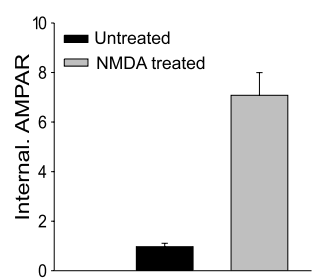

Fig. 1. NMDAR activation is sufficient to induce internalization of AMPARs. (a) Examples of internalized AMPAR immunoreactivity under different treatment conditions. Untreated cells show little AMPAR internalization, whereas cells treated with glutamate (100 $\mu \mathrm{M}$ for $1 \mathrm{~min}$ ) in the absence (middle) or presence (right) of the AMPAR antagonist CNQX $(50 \mu \mathrm{M})$ show significant staining for AMPARs internalized from the plasma membrane. (b) Q uantitation of experiments in (a) ( $n=24$ for each condition) (c) Example of untreated cell and cell treated with $50 \mu \mathrm{M}$ NMDA for $1 \mathrm{~min}$ in the presence of $C N Q X$ and MCPG (1 mM). Field widths are $0.67 \times$ those in (a). (d) Q uantitation of experiments in (c) ( $n=33-37$ for each condition).

\section{RESULTS}

\section{Activation of NMDARs alone triggers endocytosis}

Rapid internalization of AMPARs can be triggered in cultured hippocampal neurons by glutamate or the specific agonist AMPA under conditions that do not require NMDAR activation ${ }^{15,20}$. However, we also found that, in response to milder stimulus conditions, the endocytosis of AMPARs is completely blocked by $\mathrm{APV}^{20}$. This suggests either that activation of NMDARs can facilitate the internalization of AMPARs triggered by AMPAR activation, or that activation of NMDARs alone is sufficient to drive internalization. We initially investigated this latter possibility, as it has important implications for delineating the mechanisms of synaptic plasticity.

The role of NMDAR activation in triggering AMPAR endocytosis was determined using a technique described previously ${ }^{20}$, which involves staining surface AMPARs with an antibody to the AMPAR subunit GluR1, and then, following agonist treatment, stripping away surface-bound antibodies so that only internalized AMPARs are visualized (details in Methods). Treatment of cultures with glutamate ( $100 \mu \mathrm{M}$ for $1 \mathrm{~min})$ caused significant internalization of AMPARs, which still occurred when AMPARs were blocked with the AMPAR antagonist CNQX $(20 \mu \mathrm{M}$; Fig. 1a and b; untreated, $1.0 \pm 0.2, n=24$; glutamate, $7.9 \pm 1.3, n=26$; glutamate with CNQX, $4.5 \pm 1.0, n=24, p<0.05$; internalized AMPAR immunoreactivity normalized to untreated condition). Application of CNQX alone (for 10-15 min) did not cause significant AMPAR endocytosis above that observed in untreated cells (CNQX, $1.0 \pm 0.2$, $n=50$; untreated, $1.0 \pm 0.2, n=51$; data not shown), although a longer treatment (30 min) caused a slight increase in AMPAR internalization $(2.2 \pm 0.7, n=20, p<0.05)$. These results indicate that AMPAR activation is not required to trigger AMPAR endocytosis. Consistent with this conclusion, brief application of NMDA alone ( $50 \mu \mathrm{M}$ for $1 \mathrm{~min}$ ) in the presence of both AMPAR and mGluR antagonists (20 $\mu \mathrm{M}$ CNQX and $1 \mathrm{mM} \mathrm{MCPG}$, respectively) caused
Fig. 2. AMPARs internalized by AMPAR and NMDAR activation demonstrate distinct spatial localization. (a) Representative cell treated with AMPA (5 $\mathrm{min})$ shows prominent staining for internalized AMPARs in the soma and proximal dendrites. Cell treated with NMDA (1 min) shows staining in more distal dendrites. (b) Q uantitation of the percentage of total AMPAR fluorescence found in the dendrites (> $10 \mu \mathrm{m}$ from soma) following NMDA or AMPA treatment. Percentages are from cells processed either 5 or 20 min following initial application of agonist ( $n=29$ for each condition). (c, d) Q uantitation showing that the pattern of internalized AMPAR staining is stable over time. Relative fluorescence of internalized AMPARs in somatic (c) or dendritic (d) regions is shown for cells treated with AMPA or NMDA at 5 and 20 min after agonist application ( $n=32$ for each condition). a
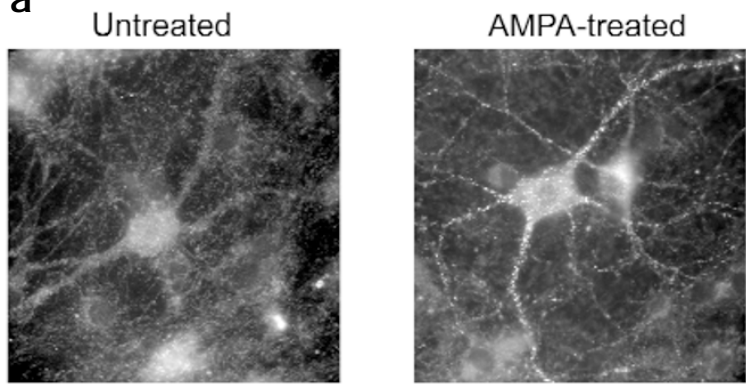

b

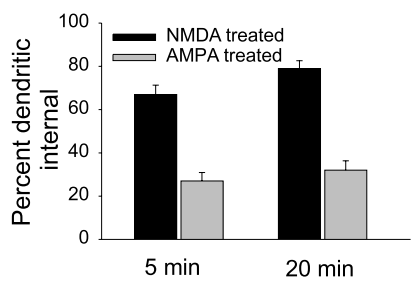

C

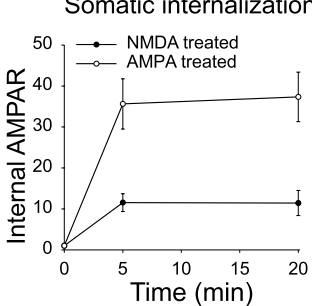

NMDA-treated

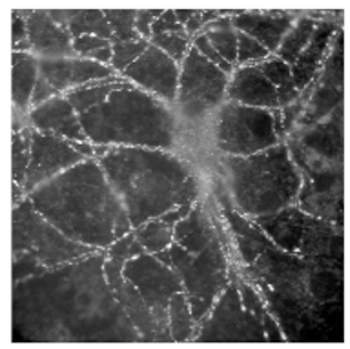

d

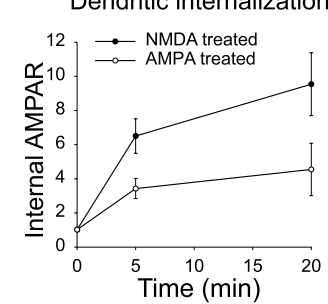




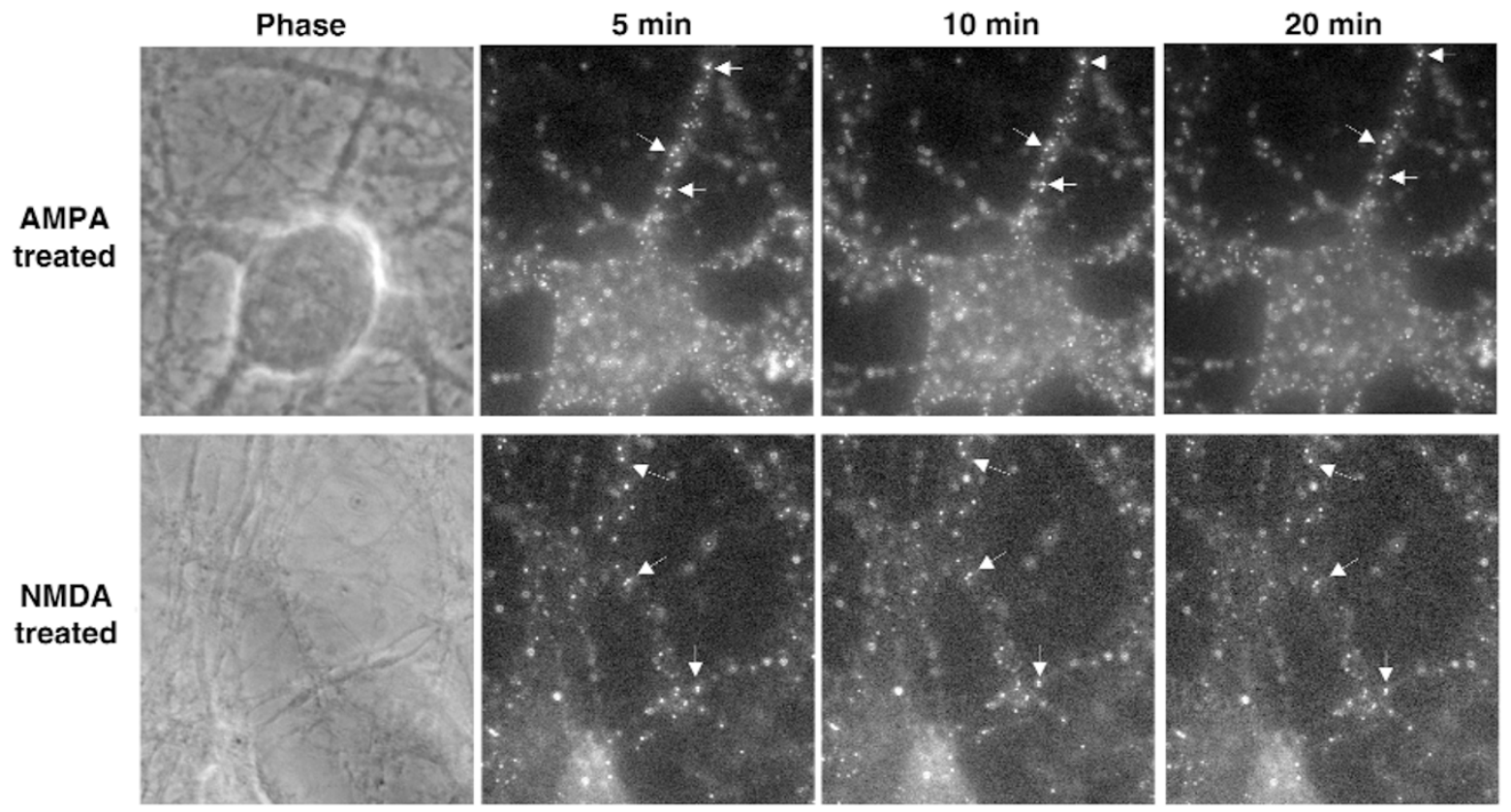

Fig. 3. Live imaging of internalized AMPARs following AMPA or N MDA treatment. Images show pattern of AMPAR staining in single cells at three time points after agonist treatment. N ote the absence of significant movement of vesicles containing AMPA Rs (see arrows).

prominent internalization of AMPARs (Fig. 1c and d; untreated, $1.0 \pm 0.1 n=37 ; \mathrm{NMDA}, 7.1 \pm 0.9, n=33, p<0.001)$. Indeed, significant AMPAR internalization was induced by exposure to NMDA for only 5-15 seconds (data not shown).

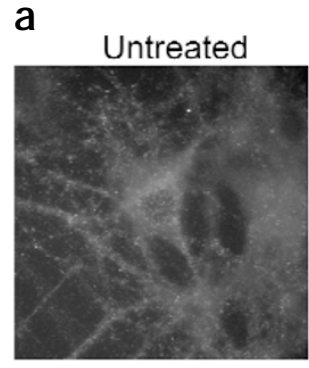

Untreated

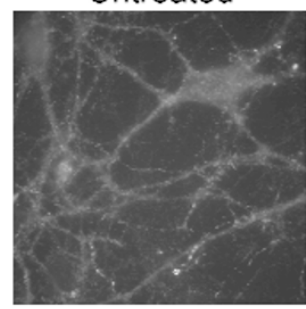

b

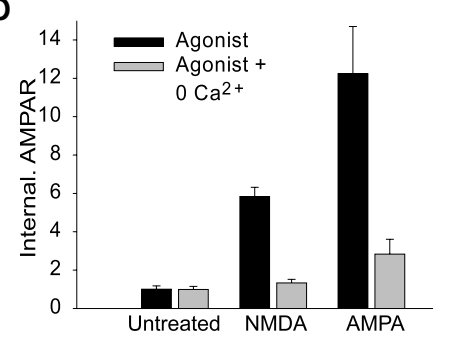

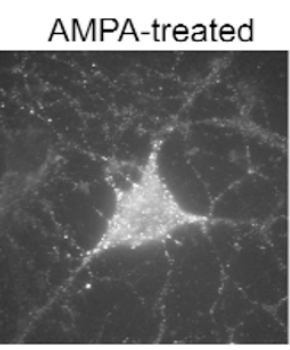

NMDA-treated

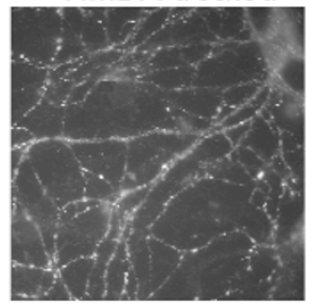

C

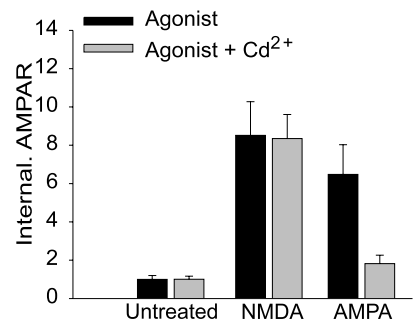

\section{Spatial localization of AMPAR endocytosis}

Following NMDA treatment, internalized AMPARs were observed in endocytic vesicles that were primarily in the more distal portions of dendrites (Figs. 1c and 2a). This is a different pattern than is observed following the activation of AMPARs alone. Following exposure of cultures to AMPA $(100 \mu \mathrm{M}$ for $5 \mathrm{~min}$ in the presence of APV and MCPG), internalized AMPARs were observed primarily in the soma and proximal dendrites (Fig. 2a). To quantify the differences in the localization of internalized AMPARs, we measured total AMPAR immunofluorescence in the dendrites ( $>10 \mu \mathrm{m}$ from soma) and compared this to the immunofluorescence detected in the soma and proximal dendrites (Methods). Twenty minutes after application of NMDA, $79 \pm 4 \%(n=29)$ of the internalized AMPAR staining was found in the dendrites, whereas only $32 \pm 5 \%(n=29)$ of the internalized AMPARs were dendritic following application of AMPA (Fig. 2b).

The difference in the localization of internalized AMPARs following activation of NMDARs versus AMPARs could be mediated by two possible mechanisms. Activation of AMPARs versus NMDARs could

Fig. 4. Both $A M P A$ - and NMDA-induced internalization of AMPARs are calcium dependent. (a) Samples of cells treated with AMPA or NMDA in the presence (middle) or absence (right) of extracellular calcium. Field widths at bottom are $1.3 \times$ those at top. (b) Q uantitation of experiments shown in (a) $(n=20-23$ for each condition). (c) Q uantitation of experiments applying AMPA or N MDA in the absence or presence of cadmium to block calcium channels ( $n=25-32$ for each condition) demonstrates that cadmium prevents AMPA - but not NMDAinduced internalization of AMPARs. 


\section{articles}

Fig. 5. Inhibition of calcineurin blocks AMPA - and N MDA-induced internalization of AMPARs. (a, b) Sample of cells treated with AMPA (a) or NMDA (b) in the absence (middle) or presence (right) of FK 506. Field widths in (b) are $2 x$ those in (a). (c) Example cells in which internalized transferrin (green) and internalized AMPARs (red) were imaged simultaneously in control (left) and NMDAtreated cells (middle). Note the lack of overlap of transferrin and AMPARs. FK-506 blocked the internalization of AMPARs but not the internalization of transferrin (right). (d, e) Q uantitation of AMPAR internalization in AMPA(d) or NMDA-treated (e) cells incubated in the presence or absence of several calcineurin inhibitors. Experiments were done in untreated cells ( $n=54$ and 62 ) or agonist-treated cells incubated without inhibitor ( $n=53$ and 90 ) or in the presence of FK-506 $(n=24$ and 35), rapamycin (an analog of FK-506 that does not inhibit calcineurin, $n=25$ and 24 ), cypermethrin $(n=34$ and 26$)$ or cyclosporin A (CsA, $n=19$ and 14$)$. a

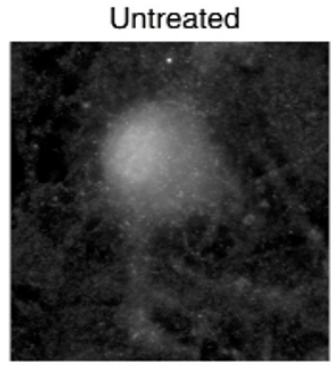

b

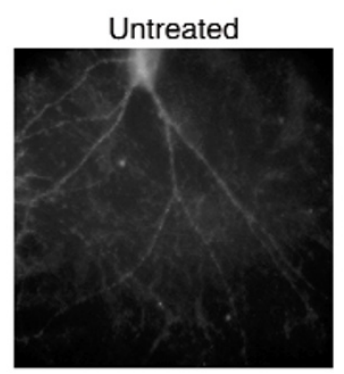

AMPA treated

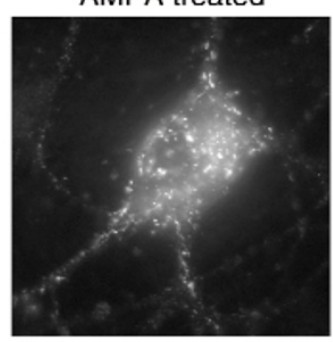

NMDA treated

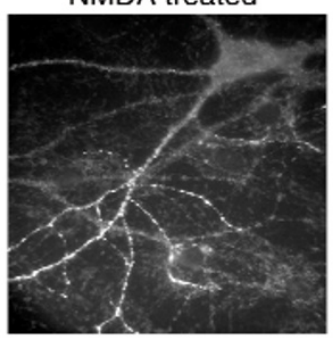

AMPA + FK-506

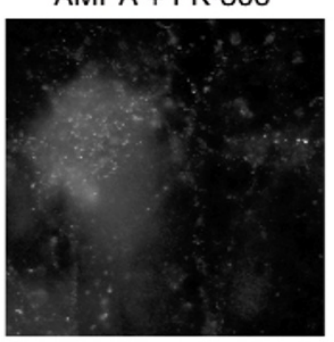

NMDA + FK-506

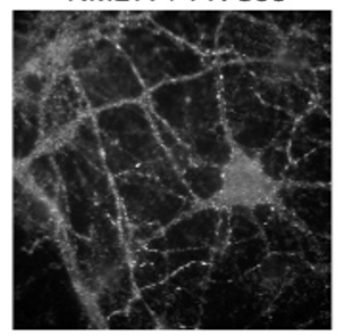

C

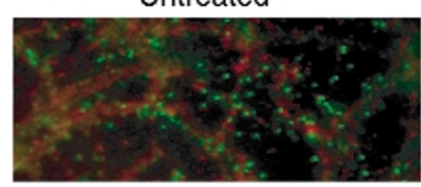

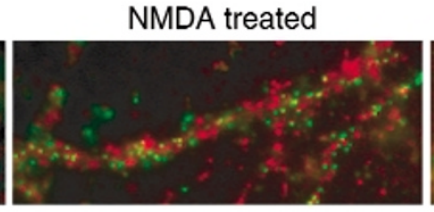

NMDA + FK-506

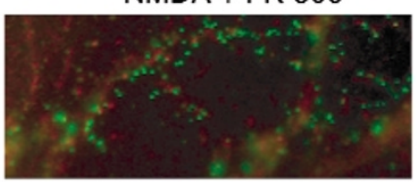

trigger different signaling pathways that regulate AMPAR trafficking in distinct regions of the neuron. Alternatively, in both cases receptors may be initially endocytosed in the dendrites, but only following activation of AMPARs might vesicles containing internalized AMPARs be trafficked toward the soma, as would be expected if the receptors were targeted for lysosomal degradation. To distinguish these possibilities, we compared the distribution of internalized AMPARs at two different intervals, 5 and 20 minutes,

following agonist exposure. In response to both AMPA and NMDA, the relative distribution of AMPAR-containing endocytic vesicles in the soma and dendrites remained largely unchanged over this time interval (Fig. 2b-d).

We next examined the possible movements of AMPAR-containing vesicles in a more dynamic fashion using time-lapse fluorescence microscopy to visualize internalized AMPARs in living cells. Live cells were fed sequentially with a rabbit antibody to GluR1 and a Cy3-conjugated donkey anti-rabbit antibody followed by treatment with NMDA (in the presence of tetrodotoxin (TTX), CNQX and MCPG) or AMPA (in the presence of TTX, APV and MCPG). After residual AMPAR surface labeling was eliminated using an abbreviated stripping procedure (10-30 s), cells were transferred to a heated microscope stage for live observation of internalized AMPARs at 5-minute intervals (Fig. 3). AMPA treatment resulted in AMPAR-containing endocytic vesicles that were localized primarily in the soma and proximal processes and exhibited little movement over a 15-minute period (Fig. 3, top row). In contrast, following NMDA treatment, most AMPAR-containing endocytic vesicles were observed in more peripheral processes (Fig. 3, bottom row), but similar to the AMPA-treated cells, these vesicles were relatively non-motile. These observations indicate that the differential distribution of internalized AMPARs observed after AMPAR versus NMDAR activation is not the result of redistribution of receptor-containing vesicles after endocytosis. Rather, it appears that the AMPARs remain relatively well localized to spatially restricted microdomains after their internalization, in contrast to the rapid anterograde and retrograde movements of vesicles containing internalized transferrin receptors ${ }^{28}$.

\section{Role of $\mathrm{Ca}^{2+}$ and calcineurin in AMPAR endocytosis}

The observation that NMDAR activation alone can trigger AMPAR endocytosis supports the hypothesis that this form of trafficking is involved in the expression of NMDAR-dependent LTD. We therefore performed a series of experiments investigating whether the signaling pathways that are necessary to induce LTD also mediate regulated AMPAR endocytosis. The earliest step required for the induction of NMDAR-dependent LTD is an elevation in postsynaptic calcium concentration ${ }^{29}$. The requirement 
for an influx of calcium in triggering AMPAR endocytosis was tested by removing extracellular calcium from the bathing medium. This was sufficient to block or strongly inhibit the internalization of AMPARs induced by either NMDA or AMPA application (Fig. 4a and b; NMDA, $5.5 \pm 0.6, n=20$; NMDA with $0 \mathrm{Ca}^{2+}, 1.4 \pm 1.2, n=20, p<0.001$; AMPA, $12.2 \pm 2.5, n=23$; AMPA with $\left.0 \mathrm{Ca}^{2+}, 2.8 \pm 0.8, n=23, p<0.001\right)$. This blockade of regulated AMPAR endocytosis is not simply due to a general inhibition of clathrin-coated pit function, as constitutive internalization of transferrin receptors (measured by uptake of fluorescently labeled transferrin), which is also mediated by clathrin-coated pits ${ }^{30}$, still occurred in the dendrites and soma after removal of extracellular calcium (data not shown).

The activation of AMPARs was done in the presence of APV; therefore NMDARs could not be the source of the calcium that is required to trigger AMPAR endocytosis under these conditions. The most likely alternate pathway for calcium influx is through voltage-dependent calcium channels that open in response to the depolarization resulting from AMPAR activation. To test this prediction, we applied agonists in the presence of the general calcium channel blocker cadmium $\left(\mathrm{Cd}^{2+}\right)$. Cadmium $(200 \mu \mathrm{M}) \mathrm{did}$ not affect NMDAR-dependent AMPAR endocytosis but strongly inhibited the internalization of AMPARs triggered by AMPA application (Fig. 4c; NMDA alone, $8.5 \pm 1.8, n=25$, NMDA with $\mathrm{Cd}^{2+}, 8.4 \pm 1.3, n=25$; AMPA alone, $6.5 \pm 1.6, n=32$, AMPA with $\left.\mathrm{Cd}^{2+}, 1.8 \pm 0.4, n=32, p<0.05\right)$. These results indicate that activation of voltage-dependent calcium channels strongly enhances AMPAR endocytosis when NMDARs are blocked but is

a
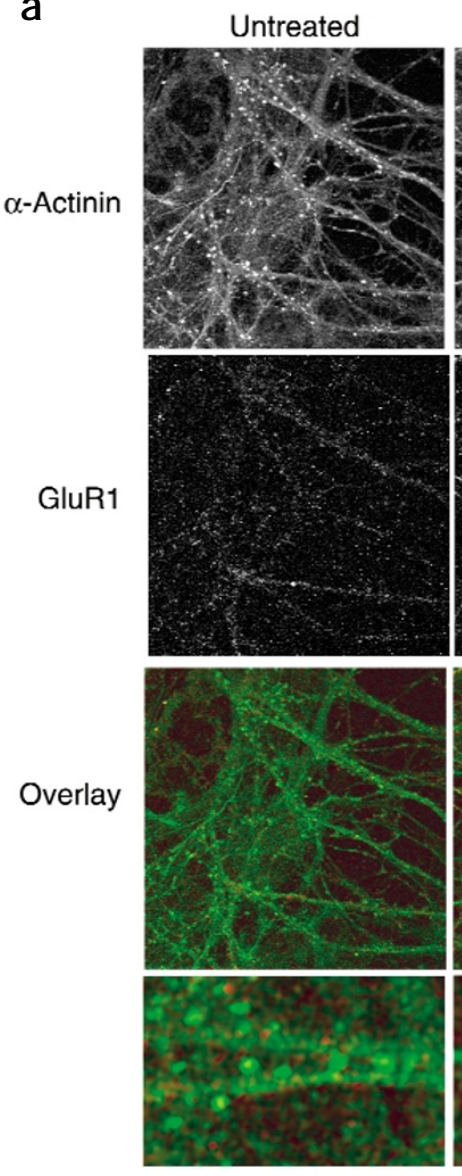

NMDA treated
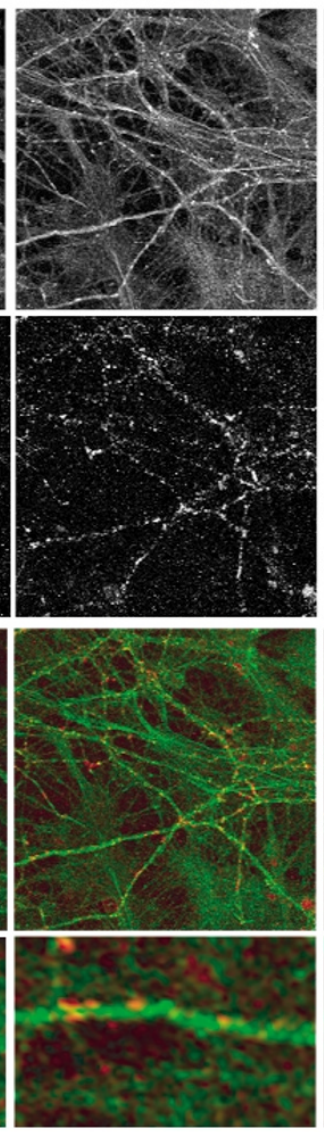

not required when calcium can directly enter the cell through the NMDAR channel.

The one calcium-sensitive effector known to be necessary for the induction of NMDAR-dependent LTD is the protein phosphatase calcineurin ${ }^{26}$. We therefore used the calcineurin inhibitor FK-506 (ref. 31) to test whether activation of this enzyme is required for the AMPAR internalization triggered by application of AMPA or NMDA. Application of FK-506 $(1 \mu \mathrm{M})$ beginning 10 minutes before ligand application strongly inhibited the endocytosis of AMPARs induced by either treatment (Fig. 5; AMPA alone, 15.9 $\pm 1.5, n=53$; AMPA with FK-506, $3.6 \pm 0.6, n=24$, $p<0.001$; NMDA alone, $17.0 \pm 2.0, n=90$; NMDA with FK-506, $5.1 \pm 1.4, n=35, p<0.001)$. In contrast, application of rapamycin, which is structurally similar to FK-506 but does not inhibit calcineurin ${ }^{31}$, did not affect the regulated AMPAR endocytosis (Fig. 5d and e; AMPA with rapamycin, $19.5 \pm 3.7, n=25$; NMDA with rapamycin, $15.5 \pm 3.5, n=24$ ). To ensure that FK506 did not simply affect general endocytosis mechanisms, we tested its effects on transferrin uptake and found that in the same cells in which AMPAR endocytosis was strongly inhibited by FK506, transferrin endocytosis was only modestly affected (Fig. 5c). Interestingly, in control cells not treated with FK-506, there was minimal overlap between the internalized AMPARs and internalized transferrin (Fig. 5c, middle), suggesting that AMPARs and transferrin receptors may be trafficked via distinct endosomal membrane compartments after endocytosis.

To further test that the inhibition of AMPAR endocytosis by FK-506 was due to inhibition of calcineurin, we examined the effects of cypermethrin and cyclosporin A, which inhibit calcineurin via mechanisms distinct from that of FK-506 (refs. 31, 32). Both of these drugs inhibited the AMPAR endocytosis induced by either NMDA or AMPA application (Fig. 5d and e; AMPA

Fig. 6. Stabilization of actin in dendritic spines does not block the N MDA-induced internalization of AMPARs. (a) Cells in which $\alpha$-actinin in dendritic spines was imaged simultaneously with internalized AMPARs following NMDA treatment in the absence (middle) or presence of jasplakinolide (right). Jasplakinolide dramatically reduced the loss of $\alpha$-actinin puncta (green) caused by N MDA treatment, but had no effect on the endocytosis of AMPARs (red). Bottom, $5 \times$ enlargement of dendritic segments shown in the overlay. (b) $Q$ uantitation of internalized AMPAR immunoreactivity from experiments shown in (a) ( $n=20$ for each condition).

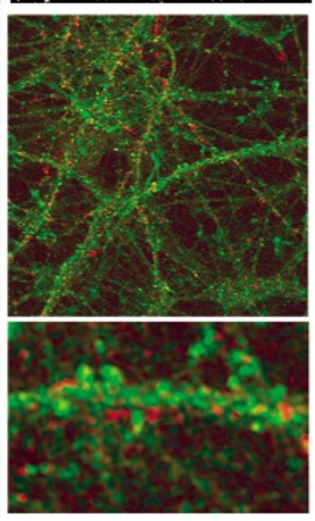

b

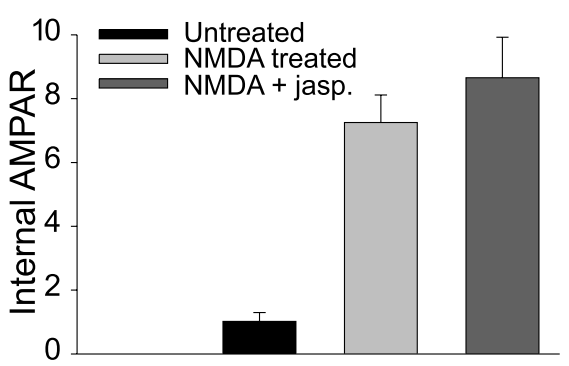


with cypermethrin, $3.0 \pm 0.7, n=34$; AMPA with cyclosporin A, $3.4 \pm 0.5, n=19$; NMDA with cypermethrin, $1.0 \pm 0.3, n=26$; NMDA with cyclosporin A, $1.2 \pm 0.4, n=14, p<0.001$ for all drug treatments versus agonist alone). These results demonstrate that like NMDAR-dependent LTD, activation of calcineurin is necessary for the AMPAR endocytosis triggered by activation of NMDARs.

Application of NMDA to cultured neurons causes a rapid loss of filamentous actin (F-actin) puncta in dendritic spines, an effect that also is blocked by inhibitors of calcineurin ${ }^{33}$. This suggests that the internalization of AMPARs following NMDA treatment may require or be a downstream consequence of actin depolymerization in dendritic spines. Consistent with these prior results, we found that application of NMDA caused a significant loss of F-actin puncta (measured by staining either with fluorescently labeled phalloidin or with an antibody to $\alpha$-actinin) as well as a significant internalization of AMPARs in the same cells (Fig. 6). To test whether these two events are causally related, we blocked loss of F-actin puncta with the actin stabilizer, jasplakinolide ${ }^{33}$. In cultures treated with jasplakinolide $(2 \mu \mathrm{M})$, NMDA treatment ( $3 \mathrm{~min}$ ) did not cause a loss of F-actin puncta, yet AMPAR internalization was unaffected (Fig. 6; NMDA, 7.3 $\pm 0.9, n=20$; NMDA with jasplakinolide, $8.7 \pm 1.3, n=20$ ). Furthermore, treatment of cells with NMDA for only 30 seconds caused AMPAR internalization but no detectable F-actin destabilization (data not shown). Thus, regulated AMPAR endocytosis does not require actin depolymerization in dendritic spines.

Inhibitors of protein phosphatase 1 (PP1) block LTD ${ }^{25-27}$, consistent with the hypothesis that the specific role of calcineurin in LTD is to dephosphorylate and consequently inactivate an endogenous inhibitor of PP1 (ref. 24). We therefore examined the effects on AMPAR endocytosis of the PP1 (and PP2A) inhibitors calyculin A and okadaic acid. Surprisingly, neither of these compounds blocked the NMDA- or AMPA-induced internalization of AMPARs. Indeed, agonist-induced internalization of AMPARs was increased in the presence of these inhibitors, which had no effect on AMPAR endocytosis when applied alone (data not shown). Interpretation of the effects of these compounds on regulated AMPAR endocytosis, however, is complicated because both inhibitors caused noticeable loss of glia in the cultures. The effects of reduced numbers of glia on the actions of NMDA or AMPA are difficult to determine, but it is conceivable that the effective concentrations of these ligands were affected.

\section{NMDA decreases mEPSC frequency}

If a significant proportion of the AMPARs that are internalized and visualized in dendrites following NMDA application were originally at synapses, as we have assumed, then NMDA application should cause a decrease in synaptic strength. To test this prediction, we recorded miniature EPSCs (mEPSCS) before and after NMDA application. Consistent with previous observations ${ }^{15}$, NMDA application caused a significant decrease in the frequency of mEPSCs (Fig. $7 ;-47 \pm 6.0 \%, n=11, p<0.05$, paired $t$-test) but a minimal reduction in their amplitude $(-13.0 \pm 5.0 \%)$. The NMDA-induced decrease in mEPSC frequency was significantly inhibited by loading cells via the patch pipette with the calcium chelator BAPTA $(26 \pm 17 \%, n=6, p<0.001)$ or with FK-506 $(-16.0 \pm 7.0 \%, n=10, p<0.05$; Fig. 7$)$ but not with okadaic acid $(-30.0 \pm 10.0, n=9)$. These results provide evidence that some proportion of the internalized AMPARs that we visualized following NMDA treatment derived originally from synapses.

Mechanisms of insulin-triggered AMPAR endocytosis

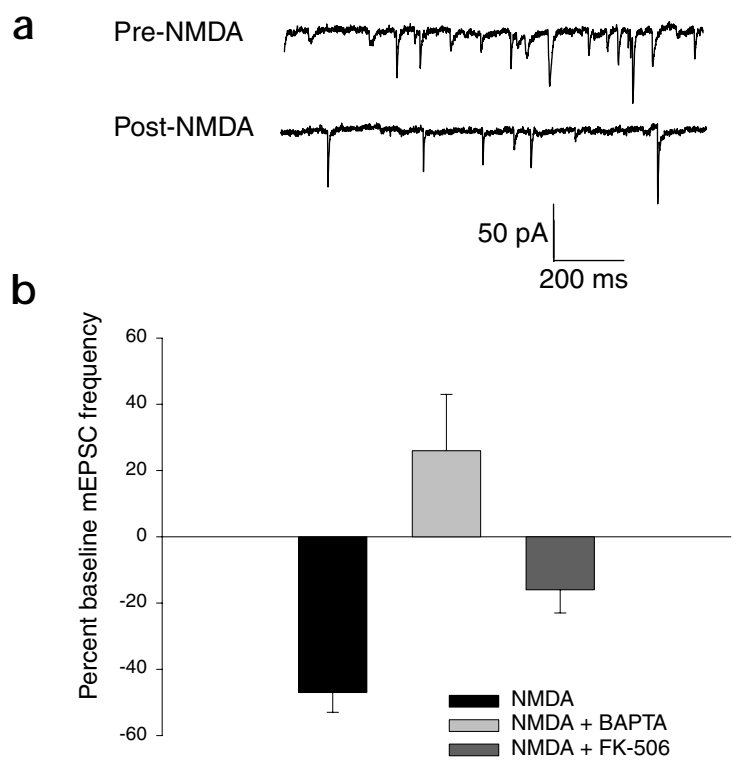

Fig. 7. The internalization of AMPARs by N MDA treatment is associated with a decreased frequency of mEPSC s. (a) Traces are examples of miniature EPSC $s$ recorded from cultured hippocampal neurons before and after the application of $20 \mu \mathrm{M} \mathrm{NMDA}$. (b) Decrease in mEPSC frequency following N MDA treatment $(n=11)$. Introduction of BAPTA $(n=6)$ or FK $-506(n=10)$ in the postsynaptic cell through the recording pipette blocked or reduced, respectively, the NMDAinduced depression of mEPSC $s$.

Treatment of hippocampal and cerebellar cultures with insulin also results in the rapid internalization of AMPARs via clathrincoated pits $^{22,23}$. It was therefore of interest to investigate whether this insulin-driven endocytosis of AMPARs shares signaling mechanisms in common with the trafficking mediated by activation of AMPARs or NMDARs. We first examined the effects of removing extracellular calcium and found that this had at most a small effect on the insulin-induced AMPAR endocytosis (Fig. 8a and c; insulin, $20.8 \pm 2.4, n=10$; insulin with $0 \mathrm{Ca}^{2+}, 15.4 \pm 1.8, n=10$ ). Similarly, application of calcineurin inhibitors had only a modest effect (Fig. 8b and d; insulin, $18.5 \pm 2.3, n=34$; insulin with FK-506, $13.8 \pm 1.5, n=45$; insulin with cypermethrin, $16.1 \pm 0.9$, $n=39$; insulin with cyclosporin A , $13.7 \pm 1.0, n=38$ ). These results provide further evidence that the removal of extracellular calcium and inhibition of calcineurin did not generally block endocytosis. Furthermore, they indicate that the triggering of AMPAR endocytosis by insulin is due to a signaling pathway different than that responsible for the endocytosis triggered by NMDAR or AMPAR activation. Consistent with this conclusion, the AMPAR endocytosis elicited by AMPA and insulin treatments was additive (AMPA, $12.4 \pm 2.7, n=6$; insulin, $18.1 \pm 7.1, n=6$; AMPA with insulin, $40.8 \pm 7.8, n=6$ ).

What signal transduction pathway does mediate the insulininduced AMPAR internalization? The downstream effects of insulin receptor activation require the activity of a series of protein kinases, including protein tyrosine kinases and protein kinase $\mathrm{C}$ $(\mathrm{PKC})^{34}$. To test whether a similar cascade might be involved in the endocytosis of AMPARs caused by insulin, we pretreated cells with the protein tyrosine kinase inhibitor genistein or the serine/threonine kinase inhibitor staurosporine. Both of these drugs strongly inhibited the insulin-induced internalization of AMPARs 
(Fig. 9a and b; insulin, 14.8 $\pm 1.7, n=28$; insulin with genistein, $1.8 \pm 0.4, n=29, p<0.001$; insulin with staurosporine, $3.0 \pm 0.8$, $n=15, p<0.001)$ yet did not block the AMPAR endocytosis caused by NMDAR or AMPAR activation (Fig. 9c). These results provide further evidence that insulin and AMPA or NMDA are acting through separate signaling pathways to promote AMPAR internalization. A further observation consistent with this conclusion is that insulin caused a more diffuse pattern of internalized AMPAR immunoreactivity than did AMPA or NMDA. This difference suggests that in addition to activating different signaling pathways, insulin and AMPA or NMDA treatment may promote differential AMPAR post-endocytic trafficking and localization.

\section{Discussion}

Previously it has been observed that AMPARs can be removed from synapses by constitutive endocytosis ${ }^{21}$ as well as regulated internalization stimulated by glutamate ${ }^{15,20}$, AMPA $^{15,20}$ or insulin ${ }^{22,23}$. Because AMPAR endocytosis seems to be an important expression mechanism for NMDAR-dependent LTD ${ }^{16,21,23}$, here we have explored the signal transduction pathways that trigger regulated AMPAR endocytosis, focusing primarily on the calcium-dependent protein phosphatase cascade that has been implicated in this form of synaptic plasticity ${ }^{24-27}$. We found that, like NMDAR-dependent LTD, activation of NMDARs can trigger AMPAR endocytosis and that this trafficking event requires calcium influx and activation of the calcium-dependent protein phosphatase calcineurin. Consistent with this endocytosis occurring at synaptic sites, we found that NMDA application caused a decrease in mEPSC frequency, a decrease that, like the AMPAR endocytosis, required a rise in calcium and calcineurin activity in the postsynaptic cells.

Activation of voltage-dependent calcium channels due to the depolarization induced by application of AMPA and the subsequent activation of calcineurin also triggers AMPAR endocytosis. This is not surprising, as activation of calcium channels can also trigger a form of LTD that shares underlying mechanisms with NMDAR-dependent LTD ${ }^{35}$. The AMPAR endocytosis induced by NMDAR or AMPAR activation, however, was not identical in that the subcellular distribution of internalized AMPARs differed, with application of NMDA causing a relatively greater proportion of punctate AMPAR endocytosis in dendrites. This observation suggests that calcium influx through dendritic, presumably synaptic, NMDARs provides a particularly effective signal for enhancing AMPAR endocytosis

Fig. 8. Insulin-induced AMPAR internalization is not blocked by removal of extracellular calcium or inhibition of calcineurin. (a) Representative cells treated with insulin in the presence (middle) or absence (right) of extracellular calcium. (b) Representative cells treated with insulin in the absence (middle) or presence (right) of FK-506. (c) Q uantitation of results from experiments shown in (a) ( $n=10-14$ for each condition). (d) Q uantitation of AMPAR internalization in insulin-treated cells incubated in the presence or absence of several calcineurin inhibitors. Experiments were done in untreated cells $(n=37)$ or agonist-treated cells incubated without inhibitor $(n=34)$ or in the presence of FK $-506(n=45)$, cypermethrin $(n=39)$ or cyclosporin $A$ ( C SA, $n=38)$. at synapses.

A surprising result was that inhibitors of PP1, which block NMDAR-dependent LTD $^{25-27}$, did not impair the endocytosis of AMPARs caused by activation of NMDARs. A similar discrepancy has been observed previously. Exposure of hippocampal slices to NMDA elicits a depression of synaptic responses that resembles NMDAR-dependent LTD $^{36}$. This 'chemLTD' is significantly inhibited by calcineurin inhibitors but is unaffected by okadaic acid or calyculin $A^{37}$. This difference in the sensitivity of chemLTD and synaptically evoked LTD to PP1/PP2A inhibitors is surprising because the two phenomena presumably share common expression mechanisms ${ }^{36}$. One explanation for these observations is that these two forms of depression have subtle differences in their triggering mechanisms or regulation. For example, exogenous application of NMDA may activate calcineurin in such a manner that it alone is sufficient to enhance AMPAR endocytosis. With modest synaptic activation of NMDARs, both PP1 and calcineurin may be necessary.

How might activation of calcineurin lead to enhanced endocytosis of AMPARs? An attractive analogy can be made to the mechanisms responsible for the endocytosis of synaptic vesicles in nerve terminals. Stimulation of nerve terminals causes a burst of clathrin-dependent synaptic vesicle endocytosis ${ }^{38,39}$. Several lines of evidence indicate that this is due to the calcineurin-mediated dephosphorylation of proteins that are part of the endocytic complex ${ }^{40-44}$. These results have led to a model that the interaction between calcineurin and dynamin 1 functions as a
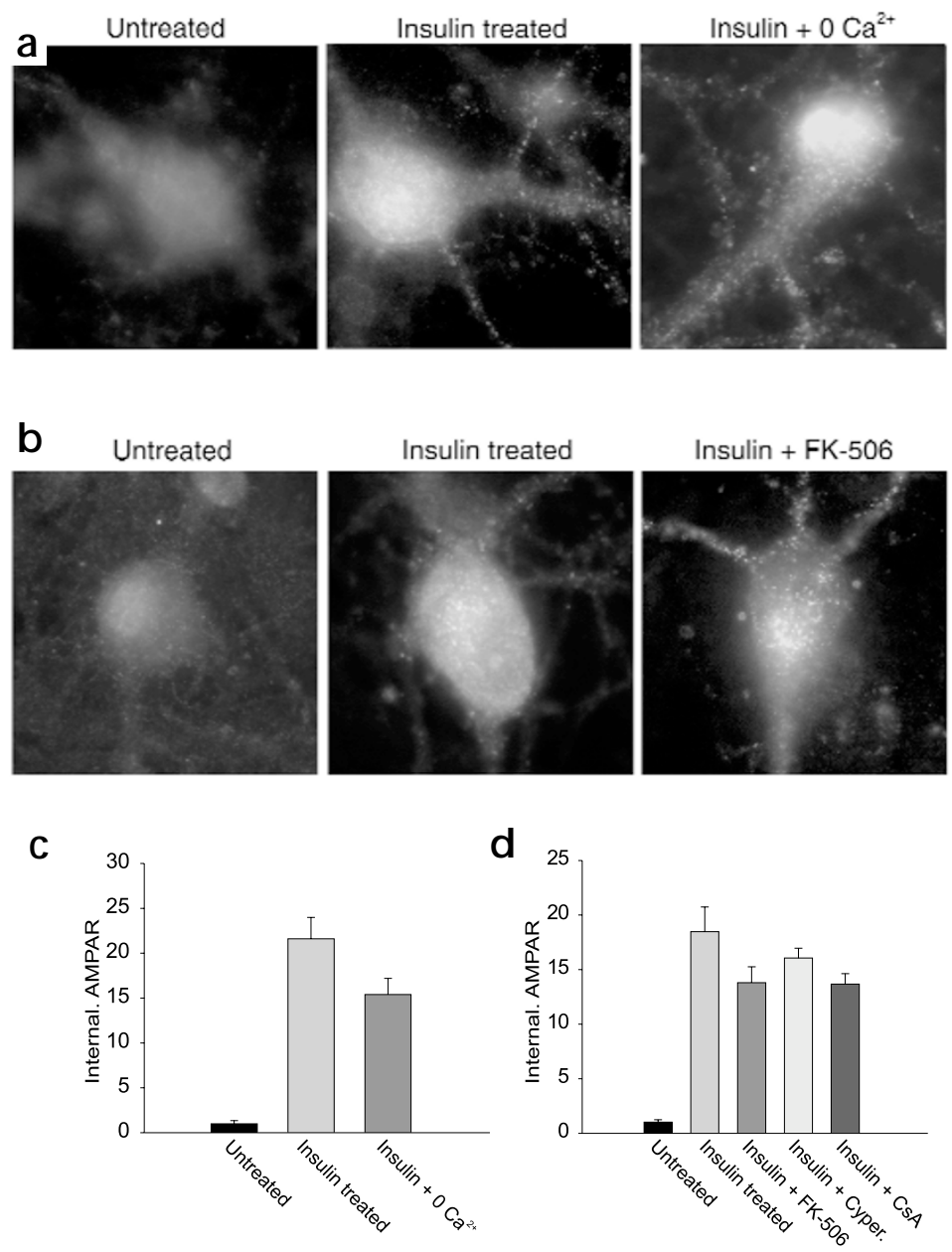
a

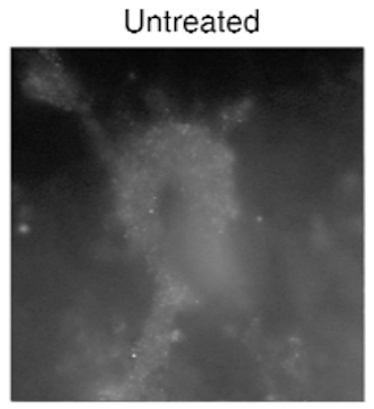

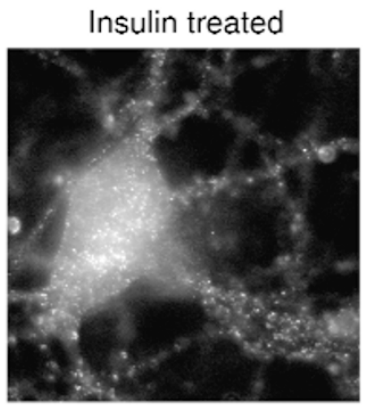

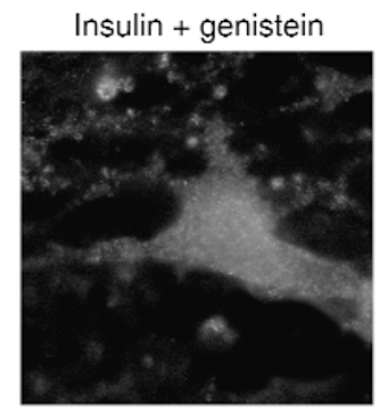

Fig. 9. Inhibition of tyrosine or serine/threonine kinases blocks insulin-induced AMPAR internalization but not that induced by AMPA or NMDA. (a) Representative cells treated with insulin in the absence of kinase inhibitors or in the presence of genistein or staurosporine. (b) Q uantitation of experiments shown in (a) $(n=15-32$ for each condition). (c) Q uantitation of effects of genistein and staurosporine on internalization of AMPARs induced by AMPA or N MDA treatment ( $n=20-25$ for each condition). b

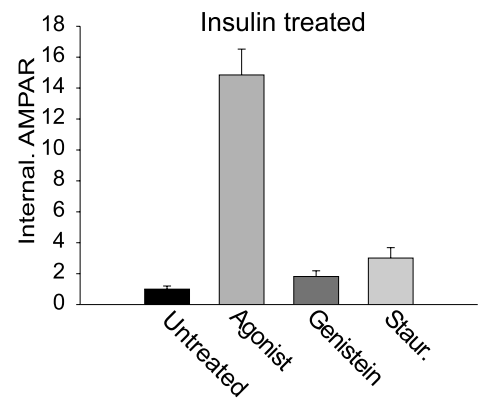

C

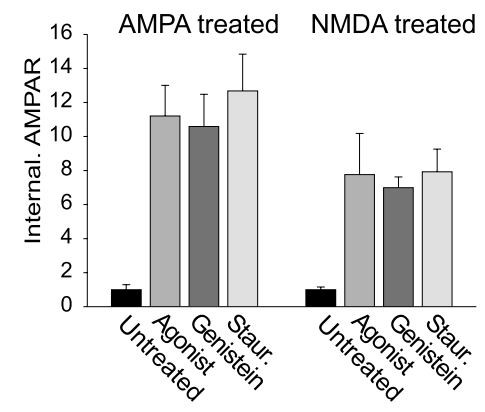

calcium sensor for endocytosis ${ }^{45}$. This complex binds to amphiphysin 1 and is then delivered to the endocytic protein complex, allowing calcineurin the proximity to dephosphorylate endocytic proteins, which in turn enhances endocytosis by promoting the assembly and function of the complex. Similar events may occur in dendritic spines following appropriate activation of NMDARs leading to the endocytosis of AMPARs and thereby LTD. Indeed, protein phosphatases other than calcineurin can contribute to the dephosphorylation of endocytic proteins ${ }^{43}$, providing a potential explanation for the additional involvement of PP1 in synaptically evoked LTD. An alternative possibility is that activation of phosphatases serves to modulate the state of AMPARs, perhaps by altering their interaction with regulatory synaptic proteins, and thereby increase their availability for endocytosis (see below).

We also examined the role of the actin cytoskeleton in AMPAR endocytosis because NMDA application causes the loss of F-actin puncta in dendritic spines ${ }^{33}$ and disruption of actin may affect the formation of clathrin-coated pits in some cell types $^{30,38}$. Application of jasplakinolide, which prevents the NMDA-induced actin breakdown, however, did not affect AMPAR endocytosis, indicating that dramatic rearrangement of the spine's actin cytoskeleton is not required for the local trafficking of endocytic vesicles that contain AMPARs.

Because insulin can elicit AMPAR endocytosis that occludes LTD $^{22,23}$, we also examined the signal transduction pathway mediating insulin's actions. The effects of insulin were minimally affected by removing extracellular calcium or inhibition of calcineurin but were almost completely blocked by a tyrosine kinase or serine/threonine kinase inhibitor. These results indicate that more than a single signaling pathway can enhance AMPAR endocytosis. Furthermore, the pattern of internalized AMPARs induced by AMPA, NMDA or insulin application differed, indicating the importance of localized signaling and trafficking mechanisms in the regulation of the surface expression of AMPARs. The potential involvement of PKC in facilitating AMPAR endo- cytosis is particularly interesting because it may be important in both cerebellar LTD ${ }^{46}$ and the LTD triggered by mGluR activation in the hippocampus ${ }^{47}$. Indeed, PKC-mediated phosphorylation of GluR2 decreases the affinity of GluR2 for the putative scaffolding protein GRIP ${ }^{48}$, a decrease that has been suggested to facilitate internalization of AMPARs ${ }^{49}$.

Several issues remain concerning the regulated endocytosis of AMPARs, including some discrepancies with another paper in this issue $^{50}$. It is likely that these differences, mostly involving the magnitude of effects, arise mainly from intentional differences in methodologies as well as from variations in culture conditions. For example, Lin et al. ${ }^{50}$ report significant levels of both constitutive and ligand-induced internalization of AMPARs in the absence of AMPAR activation. Although we recognize that constitutive internalization of AMPARs occurs ${ }^{21}$, we intentionally focused our experiments on the triggering of regulated AMPAR endocytosis and used analysis methods that subtracted off the low levels of constitutive AMPAR endocytosis. With regard to the effects of ligand binding alone on AMPAR endocytosis, we previously suggested that direct agonist binding to AMPARs, independent of changes in intracellular calcium, causes AMPAR internalization ${ }^{15}$. Consistent with this suggestion, we find here that a modest level of AMPAinduced AMPAR endocytosis still occurred even after removal of extracellular calcium and in the presence of $\mathrm{Cd}^{2+}$ or calcineurin inhibitors (Figs. 4 and 5). Furthermore, although we did not see the same degree of AMPAR internalization induced by CNQX as the accompanying paper ${ }^{50}$, prolonged treatments with this antagonist did modestly enhance AMPAR endocytosis. Finally, inhibition of calcineurin, which in our experiments strongly inhibited or blocked the AMPAR endocytosis induced by AMPA or NMDA treatment, had no effect on the AMPA-induced endocytosis of AMPARs in the experiments by Lin et al. ${ }^{50}$. This again may be partially explained by a larger contribution in their experiments of the endocytosis induced by ligand binding, an effect that may be resistant to calcineurin inhibition.

Although the differences between the two studies may be 
accounted for by methodological differences, we would also emphasize that they may, more importantly, reflect the existence of two functionally distinct populations of AMPARs at the cell surface, one of which is relatively more stabilized than the other. The non-stabilized receptors may be more susceptible to diffusion into clathrin-coated pits and subsequent internalization, a process that may be facilitated by ligand binding and does not require phosphatase activity above basal levels. Endocytosis of AMPARs that are more stabilized at the synaptic surface, perhaps because of protein-protein interactions with PDZ-domain-containing proteins, may require calcium-dependent activation of calcineurin, which would destabilize these receptors as well as facilitate the endocytic machinery ${ }^{45}$.

Two additional issues concerning the regulated endocytosis of AMPARs are worth mentioning. First, many membrane proteins that undergo constitutive endocytosis by clathrin-coated pits contain a tyrosine-based internalization motif that interacts with clathrin-associated adaptor proteins ${ }^{30}$. It is not known whether AMPAR subunits contain functionally analogous cytoplasmic sequences, what proteins may act as adaptors, and whether additional mechanism(s) are involved in mediating regulated endocytosis of AMPARs. Second, there must be great specificity in this process because the trafficking of NMDARs, which are adjacent to AMPARs in the postsynaptic plasma membrane, seems to be regulated independently from AMPARs ${ }^{15,16}$.

In summary, we have demonstrated that NMDAR activation can cause endocytosis of AMPARs and that this requires signaling mechanisms implicated in NMDAR-dependent LTD, specifically calcium influx and calcineurin activity. Constitutive transferrin receptor internalization and the AMPAR endocytosis triggered by insulin were minimally affected by manipulations that blocked the AMPAR endocytosis caused by NMDAR activation. Thus activation of calcineurin by NMDAR-mediated calcium influx seems to have a role in regulating AMPARs in the synaptic membrane. Calcium and calcineurin have also been implicated in the facilitation of synaptic vesicle endocytosis following stimulation of presynaptic terminals, likely by regulating the formation and function of the endocytic protein complex. Thus, common mechanisms may function in regulating trafficking events in both the pre- and postsynaptic membranes.

\section{Methods}

Cell culture. Hippocampi were taken from P0 rat pups, and the dentate gyri were removed. Tissue was dissociated by papain treatment followed by trituration with glass pipettes. Cells were plated on poly D-lysine-coated cover slips at a density of approximately 75,000 cells per $12 \mathrm{~mm}$ well and grown in MEM supplemented with 10\% FBS. Media was changed completely on the day after plating and in part each week thereafter. Glial growth was inhibited by FUDR after one week in culture.

Immunocytochemistry. Internalized AMPARs were selectively visualized as described ${ }^{20}$. Surface AMPARs were labeled in live neurons by $15-\mathrm{min}$ incubation with an antibody directed at the $\mathrm{N}$-terminus of the GluR1 receptor subunit (1:15 in conditioned media, Oncogene Research Products, Boston, Massachusetts). Following washout of the antibody, cells were treated with agonists as described in the text. For AMPA $(100 \mu \mathrm{M})$, glutamate $(100 \mu \mathrm{M})$ and insulin $(500 \mathrm{nM})$ treatment, cells were treated for 5 to $15 \mathrm{~min}$ in the presence of $50 \mu \mathrm{M} \mathrm{D}$-APV and $1 \mu \mathrm{M}$ TTX. NMDA $(100 \mu \mathrm{M})$ was applied for $5 \mathrm{~s}$ to $3 \mathrm{~min}$ in the presence of $20 \mu \mathrm{M}$ CNQX and $1 \mu \mathrm{M}$ TTX. Agonist was washed out, and cells were further incubated in the presence of APV and CNQX. All experiments were allowed to incubate for a total of $15 \mathrm{~min}$ at $37^{\circ} \mathrm{C}$ after initial agonist application. The presence of $1 \mathrm{mM}$ MCPG had no effect on receptor internalization, and results presented represent experiments with and without this antagonist. Following agonist application, cells were chilled on ice and surface stripped with an acidic solution (0.5 M NaCl, $0.2 \mathrm{~N}$ acetic acid) for $3 \mathrm{~min}$. Cells were then fixed in $4 \%$ PFA, permeabilized in $0.1 \%$ Triton-X100 and stained with donkey anti rabbit Cy3. Filamentous actin (F-actin) was labeled with either Alexa 488 labeled phalloidin or a monoclonal antibody against the actin binding protein $\alpha$-actinin (1:200; Chemicon, Temecula, California) following fixation and permeabilization. Secondary antibodies used in these experiments were Alexa 488 goat anti-mouse and Alexa 568 goat anti-rabbit.

To ensure that application of the bivalent GluR1 antibody itself did not stimulate AMPAR endocytosis, we applied AMPA or NMDA at $37^{\circ} \mathrm{C}$ in the absence of antibody, and then chilled cells to $4^{\circ} \mathrm{C}$ (to stop subsequent membrane trafficking) before labeling surface AMPARs with antibody and comparing the level of remaining surface AMPARs in control and treated cells. AMPA or NMDA treatment caused a clear and significant decrease in the amount of surface AMPAR immunoreactivity (AMPA, $-41.4 \pm 10.4 \%, n=26, p<0.05$; NMDA, $-43.5 \pm 8.5, n=25$, $p<0.005)$. These results confirm that NMDA and AMPA application trigger AMPAR endocytosis in the absence of bound antibody and provide an estimate of the proportion of surface AMPARs that are internalized by these treatments.

For live staining experiments, cells were labeled by a 10-min exposure to GluR1 antibody followed by $5 \mathrm{~min}$ in primary and Cy3-conjugated secondary antibody. Cells were then treated with agonist as described at $37^{\circ} \mathrm{C}$, acid-stripped for $30 \mathrm{~s}$ and immediately placed in a heated imaging chamber for observation on the microscope stage.

Enzyme inhibitors (FK-506, cypermethrin, cyclosporin A, rapamycin, staurosprine, genistein; $1 \mu \mathrm{M}$ ) were applied for 30-60 min before agonist treatment except where stated otherwise. Extracellular calcium was removed by replacing media with a nominally calcium-free media containing $10 \mathrm{mM}$ EGTA 5 min before treatment. Insulin and TTX were obtained from Sigma (St. Louis, Missouri). FK-506, cypermethrin, cyclosporin A, rapamycin, staurosporine and genistein were obtained from Calbiochem (San Diego, California). All other drugs were obtained from Tocris (Ballwin, Missouri).

Data analysis. Labeled cells were imaged using a $63 \times$ objective mounted on a Zeiss Axiovert. Images were obtained using a cooled CCD camera (Hammamatsu, Bridgewater, New Jersey) and were analyzed using Metamorph software (Universal Imaging, West Chester, Pennsylvania). For individual experiments, images for all conditions were analyzed using identical acquisition parameters. Images from each experiment were thresholded to subtract the average background fluorescence in untreated control cells. The total thresholded area of fluorescently labeled, internalized AMPARs was measured automatically by the Metamorph software and divided by the total cell area, which was determined by setting a lower threshold level to measure background fluorescence. For each experiment, internal fluorescence in all cells was normalized by dividing by the average fluorescence of untreated control cells. For the experiments comparing somatic and dendritic localization of internalized AMPARs, all images were obtained from comparable focal planes. Each experiment was repeated at least three times, and individual normalized cell values were averaged for each experimental condition. There were no differences in the results from the blind and non-blind analyses in any experiment, and therefore results of all analyses were combined. The $n$ value given for each experiment refers to the number of cells analyzed. For localization of $\alpha$-actinin, data were collected on a Microradiance confocal microscope (Biorad, Hercules, California). Statistics were done using one-way ANOVA analysis.

Electrophysiology. Cultures ( 2 to 3 weeks in vitro) were prepared exactly as for the immunocytochemistry experiments. Whole-cell patch recordings were made with an Axopatch 1D amplifier using low-resistance pipettes $(2-5 \mathrm{M} \Omega)$. The pipette solution contained $120 \mathrm{mM}$ Cs-gluconate, $8 \mathrm{mM} \mathrm{NaCl}, 10 \mathrm{mM}$ HEPES, $0.5 \mathrm{mM}$ EGTA, $2 \mathrm{mM} \mathrm{MgATP}$ and $0.3 \mathrm{mM} \mathrm{NaGTP}$, adjusted to $\mathrm{pH} 7.2$ with $\mathrm{CsOH}$. In some experiments, $10 \mathrm{mM}$ BAPTA (substituted for equimolar Cs-gluconate), FK-506 $(1 \mu \mathrm{M})$ or okadaic acid $(1 \mu \mathrm{M})$ was added to the pipette solution. The extracellular solution contained $120 \mathrm{mM} \mathrm{NaCl}, 5 \mathrm{mM} \mathrm{KCl}, 5 \mathrm{mM}$ HEPES, $20 \mathrm{mM}$ glucose, $25 \mathrm{mM}$ sucrose, $1.8 \mathrm{mM} \mathrm{CaCl}_{2}$ and $1 \mathrm{mM} \mathrm{MgCl}_{2}$, adjusted to $\mathrm{pH} 7.4$ with $\mathrm{NaOH}$. For recording miniature EPSCs (mEPSCs), $100 \mu \mathrm{M}$ 
lidocaine was added to the extracellular solution, and cells were held at $-70 \mathrm{mV}$ in voltage clamp. The series and input resistance of the cell was recorded and monitored throughout the experiment using Igor Pro software (Wavemetrics, Lake Oswego, Oregon). The mEPSCs were acquired during a 10-min baseline, after which $20 \mu \mathrm{M}$ NMDA with $20 \mu \mathrm{M}$ glycine was applied to cultures for $8 \mathrm{~min}$. Ten min after the complete washout of NMDA, 10 more min of mEPSCs were acquired for comparison to baseline. Miniature EPSCs were filtered at $1 \mathrm{kHz}$ and were digitized at $2 \mathrm{kHz}$ and analyzed using the Mini Analysis Program (Synaptosoft, Leonia, New Jersey). Threshold mEPSC amplitude was set at 5 pA.

\section{ACKNOWLEDGEMENTS}

We thank Mark Bunin for assistance in preparing the hippocampal cultures and the Mobley laboratory for the use of their confocal microscope. This work was supported by grants from NIH. X.Y. was supported by a Wellcome Travelling Research Fellowship.

\section{RECEIVED 28 SEPTEMBER; ACCEPTED 26 OCTOBER 2000}

1. Malenka, R. C. \& Nicoll, R. A. Long-term potentiation-a decade of progress? Science 285, 1870-1874 (1999).

2. Lüscher, C., Nicoll, R. A., Malenka, R. C. \& Muller, D. Synaptic plasticity and dynamic modulation of the postsynaptic membrane. Nat. Neurosci. 3, 545-550 (2000)

3. Malinow, R., Mainen, Z. F. \& Hayashi, Y. LTP mechanisms: from silence to four-lane traffic. Curr. Opin. Neurobiol. 10, 352-357 (2000).

4. Kullmann, D. M. Amplitude fluctuations of dual-component EPSCs in hippocampal pyramidal cells: implications for long-term potentiation. Neuron 12, 1111-1120 (1994).

5. Isaac, J. T., Nicoll, R. A. \& Malenka, R. C. Evidence for silent synapses: implications for the expression of LTP. Neuron 15, 427-434 (1995).

6. Liao, D., Hessler, N. A. \& Malinow, R. Activation of postsynaptically silent synapses during pairing-induced LTP in CA1 region of hippocampal slice. Nature 375, 400-404 (1995).

7. Durand, G. M., Kovalchuk, Y. \& Konnerth, A. Long-term potentiation and functional synapse induction in developing hippocampus. Nature 381, 71-75 (1996).

8. Feldman, D. E. \& Knudsen, E. I. Experience-dependent plasticity and the maturation of glutamatergic synapses. Neuron 20, 1067-1071 (1998).

9. Kullmann, D. M. \& Asztely, F. Extrasynaptic glutamate spillover in the hippocampus: evidence and implications. Trends Neurosci. 21, 8-14 (1998)

10. Choi, S., Klingauf, J. \& Tsien, R. W. Postfusional regulation of cleft glutamate concentration during LTP at 'silent synapses'. Nat. Neurosci. 3, 330-336 (2000).

11. Gasparini, S., Saviane, C., Voronin, L. L. \& Cherubini, E. Silent synapses in the developing hippocampus: Lack of functional AMPA receptors or low probability of glutamate release? Proc. Natl. Acad. Sci. USA 97, 9741-9746 (2000).

12. Rao, A. \& Craig, A. M. Activity regulates the synaptic localization of the NMDA receptor in hippocampal neurons. Neuron 19, 801-812 (1997).

13. Lissin, D. V. et al. Activity differentially regulates the surface expression of synaptic AMPA and NMDA glutamate receptors. Proc. Natl. Acad. Sci. USA 95, 7097-7102 (1998).

14. Liao, D., Zhang, X., O’Brien, R., Ehlers, M. D. \& Huganir, R. L. Regulation of morphological postsynaptic silent synapses in developing hippocampal neurons. Nat. Neurosci. 2, 37-43 (1999).

15. Lissin, D. V., Carroll, R. C., Nicoll, R. A., Malenka, R. C. \& von Zastrow, M. Rapid, activation-induced redistribution of ionotropic glutamate receptors in cultured hippocampal neurons. J. Neurosci. 19, 1263-1272 (1999).

16. Carroll, R. C., Lissin, D. V., von Zastrow, M., Nicoll, R. A. \& Malenka, R. C. Rapid redistribution of glutamate receptors contributes to long-term depression in hippocampal cultures. Nat. Neurosci. 2, 454-460 (1999).

17. Shi, S. H. et al. Rapid spine delivery and redistribution of AMPA receptors after synaptic NMDA receptor activation. Science 284, 1811-1816 (1999).

18. Hayashi, Y. et al. Driving AMPA receptors into synapses by LTP and CaMKII: requirement for GluR1 and PDZ domain interaction. Science 287, 2262-2267 (2000).

19. Lledo, P. M., Zhang, X., Sudhof, T. C., Malenka, R. C. \& Nicoll, R. A. Postsynaptic membrane fusion and long-term potentiation. Science 279, 399-403 (1998)

20. Carroll, R. C. et al. Dynamin-dependent endocytosis of ionotropic glutamate receptors. Proc. Natl. Acad. Sci. USA 96, 14112-14117 (1999).

21. Lüscher, C. et al. Role of AMPA receptor cycling in synaptic transmission and plasticity. Neuron 24, 649-658 (1999).
22. Wang, Y. T. \& Linden, D. J. Expression of cerebellar long-term depression requires postsynaptic clathrin-mediated endocytosis. Neuron 25, 635-647 (2000).

23. Man, H.-Y. et al. Regulation of AMPA receptor-mediated synaptic transmission by clathrin-dependent receptor internalization. Neuron 25, 649-662 (2000).

24. Lisman, J. A mechanism for the Hebb and the anti-Hebb processes underlying learning and memory. Proc. Natl. Acad. Sci. USA 86, 9574-9578 (1989).

25. Mulkey, R. M., Herron, C. E. \& Malenka, R. C. An essential role for protein phosphatases in hippocampal long-term depression. Science 261, 1051-1055 (1993).

26. Mulkey, R. M., Endo, S., Shenolikar, S. \& Malenka, R. C. Involvement of a calcineurin/inhibitor-1 phosphatase cascade in hippocampal long-term depression. Nature 369, 486-488 (1994).

27. O'Dell T. J. \& Kandel, E. R. Low-frequency stimulation erases LTP through an NMDA receptor-mediated activation of phosphatases. Learn. Mem. 1, 129-139 (1994).

28. Burack, M. A., Silverman, M. A. \& Banker, G. The role of selective transport in neuronal protein sorting. Neuron 26, 465-472 (2000).

29. Mulkey, R. M. \& Malenka, R. C. Mechanisms underlying induction of homosynaptic long-term depression in area CA1 of the hippocampus. Neuron 9, 967-975 (1992).

30. Schmid, S. L. Clathrin-coated vesicle formation and protein sorting: an integrated process. Annu. Rev. Biochem. 66, 511-548 (1997).

31. Liu, J. FK-506 and cyclosporin: molecular probes for studying intracellular signal transduction. Trends Pharmacol. Sci. 14, 182-188 (1993).

32. Enan, E. \& Matsumura, F. Specific inhibition of calcineurin by type II synthetic pyrethroid insecticides. Biochem. Pharmacol. 43, 1777-1784 (1992).

33. Halpain, S., Hipolito, A. \& Saffer, L. Regulation of F-actin stability in dendritic spines by glutamate receptors and calcineurin. J. Neurosci. 18, 9835-9844 (1998).

34. Heidenreich, K. A. Insulin and IGF-I receptor signaling in cultured neurons. Ann. NY Acad. Sci. 692, 72-88 (1993).

35. Cummings, J. A., Mulkey, R. M., Nicoll, R. A. \& Malenka, R. C. $\mathrm{Ca}^{2+}$ signaling requirements for long-term depression in the hippocampus. Neuron 16, 825-833 (1996).

36. Lee, H. K., Kameyama, K., Huganir, R. L. \& Bear, M. F. NMDA induces longterm synaptic depression and dephosphorylation of the GluR1 subunit of AMPA receptors in hippocampus. Neuron 21, 1151-1162 (1998).

37. Kameyama, K., Lee, H. K., Bear, M. F. \& Huganir, R. L. Involvement of a postsynaptic protein kinase A substrate in the expression of homosynaptic long-term depression. Neuron 21, 1163-1175 (1998).

38. Cremona, O. \& De Camilli, P. Synaptic vesicle endocytosis. Curr. Opin. Neurobiol. 7, 323-330 (1997).

39. Brodin, L., Low, P. \& Shupliakov, O. Sequential steps in clathrin-mediated synaptic vesicle endocytosis. Curr. Opin. Neurobiol. 10, 312-320 (2000).

40. Robinson, P. J. et al. Dynamin GTPase regulated by protein kinase C phosphorylation in nerve terminals. Nature 365, 163-166 (1993).

41. McPherson, P. S. et al. A presynaptic inositol-5-phosphatase. Nature 379, 353-357 (1996).

42. Bauerfeind, R., Takei, K. \& De Camilli, P. Amphiphysin I is associated with coated endocytic intermediates and undergoes stimulation-dependent dephosphorylation in nerve terminals. J. Biol. Chem. 272, 30984-30992 (1997).

43. Slepnev, V. I., Ochoa, G. C., Butler, M. H., Grabs, D. \& Camilli, P. D. Role of phosphorylation in regulation of the assembly of endocytic coat complexes. Science 281, 821-824 (1998).

44. Chen, H., Slepnev, V. I., Di Fiore, P. P. \& De Camilli, P. The interaction of epsin and Eps15 with the clathrin adaptor AP-2 is inhibited by mitotic phosphorylation and enhanced by stimulation-dependent dephosphorylation in nerve terminals. J. Biol. Chem. 274, 3257-3260 (1999).

45. Lai, M. M. et al. The calcineurin-dynamin 1 complex as a calcium sensor for synaptic vesicle endocytosis. J. Biol. Chem. 274, 25963-25966 (1999).

46. De Zeeuw, C. I. et al. Expression of a protein kinase C inhibitor in Purkinje cells blocks cerebellar LTD and adaptation of the vestibulo-ocular reflex. Neuron 20, 495-508 (1998).

47. Oliet, S. H., Malenka, R. C. \& Nicoll, R. A. Two distinct forms of long-term depression coexist in CA1 hippocampal pyramidal cells. Neuron 18, 969-982 (1997).

48. Matsuda, S., Mikawa, S. \& Hirai, H. Phosphorylation of serine-880 in GluR2 by protein kinase $\mathrm{C}$ prevents its $\mathrm{C}$ terminus from binding with glutamate receptor-interacting protein. J. Neurochem. 73, 1765-1768 (1999).

49. Matsuda, S., Launey, T., Mikawa, S. \& Hirai, H. Disruption of AMPA receptor GluR2 clusters following long-term depression induction in cerebellar Purkinje neurons. EMBO J. 19, 2765-2774 (2000).

50. Lin, J. W. et al. Distinct molecular mechanisms and divergent endocytotic pathways of AMPA receptor internalization. Nat. Neurosci. 3, AAA-BBB (2000). 